\title{
Invariant Sets for Constrained Nonlinear Discrete-time Systems with Application to Feasibility in Model Predictive Control
}

\author{
Eric C. Kerrigan and Jan M. Maciejowski \\ Control Group, Department of Engineering, University of Cambridge \\ Trumpington Street, Cambridge CB2 1PZ, United Kingdom, Tel: +44-1223-339222, Fax: +44-1223-332662 \\ eck21, jmm@eng.cam.ac.uk, http://www-control.eng.cam.ac.uk/
}

\begin{abstract}
An understanding of invariant set theory is essential in the design of controllers for constrained systems, since state and control constraints can be satisfied if and only if the initial state belongs to a positively invariant set for the closed-loop system. The paper briefly reviews some concepts in invariant set theory and shows that the various sets can be computed using a single recursive algorithm. The ideas presented in the first part of the paper are applied to the fundamental design goal of guaranteeing feasibility in predictive control. New necessary and sufficient conditions based on the control horizon, prediction horizon and terminal constraint set are given in order to guarantee that the predictive control problem will be feasible for all time, given any feasible initial state.
\end{abstract}

Keywords: invariant sets, positively invariant sets, control invariant sets, stabilisable sets, controllable sets, predictive control, feasibility.

\section{Introduction}

Most systems are subject to state and control constraints and the design of controllers for such systems is a very active area of research. In particular, invariant set theory [3] has been shown to be crucial in understanding the behaviour of constrained systems, since constraints can be satisfied if and only if the initial state is contained in a set which is positively invariant for the closed-loop system. Section 2 provides a unified framework for capturing the most important ideas in invariant set theory.

Invariant set theory has been very successful in providing sufficient nominal and robust feasibility and stability conditions in Model Predictive Control (MPC) [11]. One of the primary reasons for MPC's success is the ease with which constraints on both the control inputs and states can be incorporated in the controller synthesis. However, there still seems to be a few "missing links" in the understanding of the effect of the various design parameters on the feasible domain of an MPC scheme. The main aim of Section 3 is to apply the invariant set framework of Section 2 to MPC, generalise some existing results and present some new necessary and sufficient conditions for the analysis and synthesis of MPC controllers with guaranteed nominal feasibility. It is hoped that the formal framework presented in this paper will facilitate a better understanding of the most basic problem in the design of MPC controllers.

$\Omega$ is used to denote any arbitrary subset of $\mathbb{R}^{n}$. Given $\Omega, \Omega^{\circ}$ denotes its interior. The set $A \backslash B$ is the complement of $B$ that is contained in $A$, i.e. $A \backslash B \triangleq\{x: x \in A, x \notin B\}=A \cap B^{c}$. The notation $A \subseteq B$ is used to denote that $A$ is a subset of $B$ and $A \subset B$ denotes that $A$ is a proper subset of $B$. $\mathbb{Z}$ is the set of integers, $\mathbb{N}$ is the set of non-negative integers and
$\mathbb{N}_{+}$is the set of positive integers. A sequence of states or control inputs is denoted by $\left\{x_{k}\right\}_{0}^{P} \triangleq\left\{x_{0}, x_{1}, \ldots, x_{P}\right\}$. The notation $\left\{x_{k} \in \Omega\right\}_{0}^{P}$ is used to indicate that each element of the sequence $\left\{x_{k}\right\}_{0}^{P}$ is an element of $\Omega$.

\section{Invariant Set Theory}

Consider the following discrete-time, nonlinear, timeinvariant (NLTI) dynamic system:

$$
x_{k+1}=f\left(x_{k}, u_{k}\right)
$$

where $k \in \mathbb{Z}, x_{k}$ is the system state and $u_{k}$ is the control input. It is assumed that $f\left(x_{k}, u_{k}\right)$ is uniquely defined over $\mathbb{X} \times \mathbb{U}$ with $f(0,0)=0$. Full state measurement and no disturbances or model uncertainty is assumed.

The system is subject to pointwise-in-time constraints on the control inputs and/or the states:

$$
\begin{aligned}
& u_{k} \in \mathbb{U} \subset \mathbb{R}^{m} \\
& x_{k} \in \mathbb{X} \subseteq \mathbb{R}^{n}
\end{aligned}
$$

The set $\mathbb{U}$ is assumed to be compact and simply connected, while $\mathbb{X}$ is assumed to be closed and simply connected. It is assumed that $(0,0) \in \mathbb{X}^{\circ} \times \mathbb{U}^{\circ}$. An admissible control input, sequence or law is one that satisfies the input constraints. From this point on, it is understood that the control law and states are subject to the constraints in (2).

\subsection{Invariant and Positively Invariant Sets}

Definition 2.1 (Positively invariant set [3]). The nonempty set $\Omega \subset \mathbb{R}^{n}$ is positively invariant for the autonomous system $x_{k+1}=f\left(x_{k}\right)$ if and only if $\forall x_{0} \in \Omega$, the system evolution satisfies $x_{k} \in \Omega, \forall k \in \mathbb{N}_{+}$. The set $\Omega$ is invariant if and only if $x_{0} \in \Omega$ implies that $x_{k} \in \Omega, \forall k \in \mathbb{Z}$.

In general, a given set $\Omega$ is not positively invariant. However, often one would like to determine the largest positively invariant set contained in $\Omega$ :

Definition 2.2 (Maximal positively invariant set [7]).

The non-empty set $O_{\infty}(\Omega)$ is the maximal positively invariant set contained in $\Omega$ for the autonomous system $x_{k+1}=f\left(x_{k}\right)$ if and only if $O_{\infty}(\Omega)$ is positively invariant and contains all positively invariant sets contained in $\Omega$, i.e. $\Psi$ is positively invariant only if $\Psi \subseteq O_{\infty}(\Omega) \subseteq \Omega$.

Definition 2.3. The maximal positively invariant set for the closed-loop system ${ }^{1} x_{k+1}=f\left(x_{k}, g\left(x_{k}\right)\right)$ is denoted by

\footnotetext{
${ }^{1}$ In this paper statements regarding autonomous systems also apply to closed-loop systems. A superscript will denote that the associated system is a closed-loop system and that the control law is required to satisfy the input constraints.
} 
$O_{\infty}^{g}(\Omega)$ and is defined as the maximal positively invariant set contained in $\Omega^{g}$, where

$$
\Omega^{g} \triangleq\left\{x_{k} \in \Omega: g\left(x_{k}\right) \in \mathbb{U}\right\}
$$

is the input admissible subset of $\Omega$, i.e. the subset in which the control law satisfies the input constraints.

\subsection{Control Invariant Sets}

Definition 2.4 (Control invariant set [3]). The non-empty set $\Omega \subset \mathbb{R}^{n}$ is a control invariant set for the system $x_{k+1}=$ $f\left(x_{k}, u_{k}\right)$ if and only if there exists a feedback control law $u_{k}=g\left(x_{k}\right)$ such that $\Omega$ is a positively invariant set for the closed-loop system $x_{k+1}=f\left(x_{k}, g\left(x_{k}\right)\right)$ and $u_{k} \in \mathbb{U}, \forall x_{k} \in \Omega$.

In general, a given set $\Omega$ is not control invariant. However, often one would like to determine the largest control invariant set contained in $\Omega$ :

Definition 2.5 (Maximal control invariant set [2]). The non-empty set $C_{\infty}(\Omega)$ is the maximal control invariant set contained in $\Omega$ for the system $x_{k+1}=f\left(x_{k}, u_{k}\right)$ if and only if $C_{\infty}(\Omega)$ is control invariant and contains all control invariant sets contained in $\Omega$, i.e. $\Psi$ is control invariant only if $\Psi \subseteq C_{\infty}(\Omega) \subseteq \Omega$.

Theorem 2.1 ([3]). Given the NLTI system (1), the constraints (2) can be satisfied for all time $k \in \mathbb{N}$ if and only if the initial state $x_{0} \in \mathcal{C}_{\infty}(\mathbb{X}) \subseteq \mathbb{X}$.

Remark 2.1. A similar condition holds for autonomous systems and the corresponding maximal positively invariant set.

Definition 2.6 (The set $Q(\Omega)[2,6]$ ). The non-empty set $Q(\Omega)$ is defined as the set of states in $\mathbb{R}^{n}$ for which an admissible control input exists which will drive the system to $\Omega$ in one step, i.e.

$$
Q(\Omega) \triangleq\left\{x_{k} \in \mathbb{R}^{n}: \exists u_{k} \in \mathbb{U} \text { such that } f\left(x_{k}, u_{k}\right) \in \Omega\right\} .
$$

For autonomous/closed-loop systems, $Q(\Omega)$ is the set of states from which the system will evolve to $\Omega$ at the next time instant, i.e. $Q(\Omega)=\left\{x_{k} \in \mathbb{R}^{n}: x_{k+1}=f\left(x_{k}\right) \in \Omega\right\}$.

The following is a well-known geometric condition for a set to be control invariant and is often used in the derivation of properties of the various invariant sets:

Theorem 2.2 (Geometric condition for invariance [6]). The set $\Omega \subset \mathbb{R}^{n}$ is a control/positively invariant set if and only if $\Omega \subseteq Q(\Omega)$.

The set $Q(\Omega)$ is the orthogonal projection of the set $\left\{\left(x_{k}, u_{k}\right) \in \mathbb{R}^{n} \times \mathbb{R}^{m}: f\left(x_{k}, u_{k}\right) \in \Omega, u_{k} \in \mathbb{U}\right\}$ onto the first coordinate. If one has an algorithm which can calculate $Q(\Omega)$ as well as a routine for testing set inclusion, one can use this geometric condition to test whether a given set $\Omega$ is control/positively invariant. For LTI systems, with $\Omega$ and $\mathbb{U}$ given by linear inequalities, projection can be implemented using Fourier elimination [8] and $A \subseteq B$ is true if and only if all the linear inequalities in $B$ are redundant with respect to the inequalities in $A$. Additionally, $A=B$ if and only if $A \subseteq B$ and $B \subseteq A$. See [3, Sect. 4.2] for alternative invariance and subset tests.
Proposition 2.1 ([14]). The set $Q(\Omega)$ has the following properties: (i) For all $\Omega, Q(\Omega) \cap \Omega \subseteq \Omega$; (ii) For all $\Omega_{1}, \Omega_{2}$ with $\Omega_{1} \subseteq \Omega_{2}, Q\left(\Omega_{1}\right) \subseteq Q\left(\Omega_{2}\right)$; (iii) $A$ set $\Omega$ is control/positively invariant if and only if $Q(\Omega) \cap \Omega=\Omega$.

\subsection{Stabilisable Sets}

Definition 2.7 (Stabilisable set [1]). The set $\mathcal{S}_{i}(\Omega, \mathbb{T})$ is the $i$-step stabilisable set contained in $\Omega$ for the system $x_{k+1}=$ $f\left(x_{k}, u_{k}\right)$ if and only if $\mathbb{T}$ is a control invariant subset of $\Omega$ and $S_{i}(\Omega, \mathbb{T})$ contains all states in $\Omega$ for which there exists an admissible control sequence of length $i$ which will drive the state of the system to $\mathbb{T}$ in $i$ steps or less, while keeping the evolution of the state inside $\Omega$, i.e.

$$
\begin{gathered}
\mathcal{S}_{i}(\Omega, \mathbb{T}) \triangleq\left\{x_{0} \in \Omega: \exists\left\{u_{k} \in \mathbb{U}\right\}_{0}^{i-1}, \exists N \leq i\right. \text { such that } \\
\left.\left\{x_{k} \in \Omega\right\}_{1}^{N-1} \text { and }\left\{x_{i} \in \mathbb{T} \subseteq \Omega\right\}_{N}^{i}, \mathbb{T} \subseteq Q(\mathbb{T})\right\}
\end{gathered}
$$

Definition 2.8 (Maximal stabilisable set). $S_{\infty}(\Omega, \mathbb{T})$ is the maximal stabilisable set contained in $\Omega$ for the system $x_{k+1}=f\left(x_{k}, u_{k}\right)$ if and only if $S_{\infty}(\Omega, \mathbb{T})$ is the union of all $i$-step stabilisable sets contained in $\Omega$.

Remark 2.2. In general, the maximal stabilisable set $S_{\infty}(\Omega, \mathbb{T})$ is not equal to the maximal control invariant set $C_{\infty}(\Omega)$, even for linear systems: $\mathcal{S}_{\infty}(\Omega, \mathbb{T}) \subseteq C_{\infty}(\Omega)$ for all control invariant $\mathbb{T}$. The set $C_{\infty}(\Omega) \backslash S_{\infty}(\Omega, \mathbb{T})$ includes all initial states from which it is not possible to drive the system to the stabilisable region $S_{\infty}(\Omega, \mathbb{T}$ ) (and hence to $\mathbb{T}$ ). It might only be possible to bound the norm of the states $\left\|x_{k}\right\|$ as in the case of a limit cycle or to drive the system to an alternative stable equilibrium.

Remark 2.3. If $\mathbb{T}_{1} \neq \mathbb{T}_{2}$ are two control invariant sets, then $S_{\infty}\left(\Omega, \mathbb{T}_{1}\right)$ and $\mathcal{S}_{\infty}\left(\Omega, \mathbb{T}_{2}\right)$ are not necessarily equal. Similarly, $\mathcal{S}_{\infty}(\Omega,\{0\})$ is not necessarily equal to $\mathcal{S}_{\infty}(\Omega, \mathbb{T})$, since it is not always possible to drive some systems to the origin ${ }^{2}$.

\subsection{Principles for Computing Invariant Sets}

Definition 2.9 (Controllable set). The i-step controllable set $\mathcal{K}_{i}(\Omega, \mathbb{T})$ is the set of states in $\Omega$ which can be driven by an admissible input sequence of length $i$ to an arbitrary terminal set $\mathbb{T} \subset \mathbb{R}^{n}$ in exactly $i$ steps, while keeping the evolution of the state inside $\Omega$ for the first $i-1$ steps, i.e.

$$
\begin{aligned}
\mathcal{K}_{i}(\Omega, \mathbb{T}) \triangleq\left\{x_{0} \in \Omega: \exists\left\{u_{k} \in \mathbb{U}\right\}_{0}^{i-1}\right. \text { such that } \\
\\
\left.\left.\qquad x_{k} \in \Omega\right\}_{1}^{i-1} \text { and } x_{i} \in \mathbb{T} \subset \mathbb{R}^{n}\right\} .
\end{aligned}
$$

The limit, if it exists, defines the infinite-time controllable set:

$$
\mathcal{K}_{\infty}(\Omega, \mathbb{T}) \triangleq \lim _{i \rightarrow \infty} \mathcal{K}_{i}(\Omega, \mathbb{T})
$$

Remark 2.4. Note that if the notation $S_{i}(\Omega, \mathbb{T})$ is used, $\mathbb{T}$ is a control invariant subset of $\Omega$. If $\mathcal{K}_{i}(\Omega, \mathbb{T})$ is used, $\mathbb{T}$ can be any arbitrary subset of $\mathbb{R}^{n}$.

${ }^{2}$ The region $\mathcal{S}_{\infty}\left(\mathbb{R}^{n},\{0\}\right)$ can be seen to be the generalisation to nonlinear systems of the ANCBI (asymptotically null-controllable with bounded inputs) region for LTI systems with no state constraints [4]. The maximal stabilisable set $\mathcal{S}_{\infty}(\mathbb{X},\{0\})$ is a generalisation to nonlinear systems of the maximal admissible set defined in [8] and the feasible region of the predictive control scheme defined in [12]. Care has to be taken not to confuse definitions by other authors with those given in this paper. 
Remark 2.5. The one-step controllable set to $\mathbb{T}$ is equal to the intersection of $Q(\mathbb{T})$ and $\Omega$.

Proposition 2.2. Some properties of controllable sets are: (i) If $\mathcal{K}_{i}(\Omega, \mathbb{T})$ is control invariant, then so is $\mathcal{K}_{i+1}(\Omega, \mathbb{T})$. In general, the reverse statement does not hold;

(ii) If the state $x_{k} \in \mathcal{K}_{i+1}(\Omega, \mathbb{T}) \backslash \mathcal{K}_{i}(\Omega, \mathbb{T}) \neq \emptyset$, then there exists an admissible control input which will ensure that the state at the next time instant is in $\mathcal{K}_{i}(\Omega, \mathbb{T})$;

(iii) If the state $x_{k} \in \mathcal{K}_{i}(\Omega, \mathbb{T}) \backslash \mathcal{K}_{i+1}(\Omega, \mathbb{T}) \neq \emptyset$, then there does not exist an admissible control input which will ensure that the state at the next time instant is in $\mathcal{K}_{i}(\Omega, \mathbb{T})$;

(iv) If the state $x_{k} \in \mathcal{K}_{i+1}(\Omega, \mathbb{T}) \backslash \bigcup_{k=0}^{i} \mathcal{K}_{k}(\Omega, \mathbb{T}) \neq \emptyset$, then there does not exist an admissible control sequence which will drive the system to $\mathbb{T}$ in $i$ steps or less.

Definition 2.10 (Finitely determined set [7]). The set $\mathcal{K}_{\infty}(\Omega, \mathbb{T})$ is finitely determined if and only if $\exists i \in \mathbb{N}$ such that $\mathcal{K}_{\infty}(\Omega, \mathbb{T})=\mathcal{K}_{i}(\Omega, \mathbb{T})$. The smallest element $i^{*} \in \mathbb{N}$ such that $\mathcal{K}_{\infty}(\Omega, \mathbb{T})=\mathcal{L}_{i^{*}}(\Omega, \mathbb{T})$ is called the determinedness index.

In general, $\mathcal{K}_{\infty}(\Omega, \mathbb{T})$ is not finitely determined, however:

Lemma 2.1. If $\exists i \in \mathbb{N}$ such that $\mathcal{K}_{i}(\Omega, \mathbb{T})=\mathcal{K}_{i+1}(\Omega, \mathbb{T})$ then $\mathcal{K}_{\infty}(\Omega, \mathbb{T})$ is finitely determined and control invariant.

It follows that $\mathcal{K}_{i}(\Omega, \mathbb{T})=\mathcal{K}_{\infty}(\Omega, \mathbb{T}), \forall i \geq i^{*}$.

Algorithm 2.1 (Controllable sets). The controllable sets of a system can be computed via the following iterative procedure:

$$
\begin{aligned}
& \mathcal{K}_{0}(\Omega, \mathbb{T})=\mathbb{T} \\
& \mathcal{K}_{i+1}(\Omega, \mathbb{T})=Q\left(\mathcal{K}_{i}(\Omega, \mathbb{T})\right) \cap \Omega
\end{aligned}
$$

If $\mathcal{K}_{i}(\Omega, \mathbb{T})=\mathcal{K}_{i+1}(\Omega, \mathbb{T})$, then terminate the algorithm.

The basic procedure for implementing Algorithm 2.1 is: (i) compute $Q(\cdot)$; (ii) compute $Q(\cdot) \cap \Omega$ and (iii) test for set equality. These three operations are easily implemented for LTI systems subject to linear inequality constraints $[2,6,7,8,10,14]$. Though the algorithms presented in this paper are difficult to implement for general nonlinear systems, there exist some classes of nonlinear systems for which the building blocks already are in place, such as piecewise affine systems and some classes of hybrid systems [1]. Some work on developing algorithms for computing robust control invariant sets for hybrid systems has also been carried out by the authors of [14]. Set:

\subsubsection{Computing the Maximal Control Invariant}

Definition 2.11 (Admissible set [6]). The $i$-step admissible set $C_{i}(\Omega)$ contained in $\Omega$ is the set of states for which an admissible control sequence of length $i$ exists while keeping the evolution of the state inside $\Omega$ for $i$ steps, i.e.

$$
\mathcal{C}_{i}(\Omega) \triangleq\left\{x_{0} \in \Omega: \exists\left\{u_{k} \in \mathbb{U}\right\}_{0}^{i-1}:\left\{x_{k} \in \Omega\right\}_{1}^{i}\right\} .
$$

Algorithm 2.2 (Maximal control invariant set). The i-step admissible set $\mathcal{C}_{i}(\Omega)$ can be computed using Algorithm 2.1 by noting that

$$
\mathcal{C}_{i}(\Omega)=\mathcal{K}_{i}(\Omega, \Omega) \text {. }
$$

If $\mathcal{C}_{i}(\Omega)=\mathcal{C}_{i+1}(\Omega)$, then $\mathcal{C}_{\infty}(\Omega)=\mathcal{C}_{i}(\Omega)$.
Proposition 2.3. Some properties of admissible sets are:

(i) Each set $\mathcal{C}_{i+1}(\Omega) \subseteq \mathcal{C}_{i}(\Omega)$;

(ii) Each $\operatorname{set} \mathcal{C}_{i}(\Omega)=\bigcap_{k=0}^{i} \mathcal{C}_{k}(\Omega)$;

(iii) If the state $x_{k} \in \Omega \backslash C_{i}(\Omega) \neq \emptyset$ then there does not exist a control sequence which will ensure that the state evolution remains within $\Omega$ for $i$ steps;

(iv) If the state $x_{k} \in \mathcal{C}_{i}(\Omega) \backslash \mathcal{C}_{\infty}(\Omega) \neq \emptyset$ then there does not exist a control input which will ensure that the state at the next time instant is in $C_{i}(\Omega)$.

A necessary and sufficient condition for the finitedeterminedness of the maximal control invariant set is:

Theorem 2.3. $\mathcal{C}_{\infty}(\Omega)$ is finitely determined if and only if $\exists i \in \mathbb{N}$ such that $\mathcal{C}_{i}(\Omega)=\mathcal{C}_{i+1}(\Omega)$.

The following sets are defined for closed-loop systems:

Definition 2.12 (The set $\mathcal{K} O_{i}^{g}(\Omega, \mathbb{T})$ ). The set $\mathcal{K} O_{i}^{g}(\Omega, \mathbb{T})$ for the system $x_{k+1}=f\left(x_{k}, u_{k}\right)$, in closed-loop with the control law $u_{k}=g\left(x_{k}\right)$, is defined as $\mathcal{K} O_{i}^{g}(\Omega, \mathbb{T}) \triangleq \mathcal{K}_{i}\left(\Omega^{g}, \mathbb{T}\right)$ for the autonomous system $x_{k+1}=f\left(x_{k}, g\left(x_{k}\right)\right)$.

Definition 2.13 (The set $O_{i}^{g}(\Omega)$ ). The $i$-step admissible set for the system $x_{k+1}=f\left(x_{k}, u_{k}\right)$, in closed-loop with the control law $u_{k}=g\left(x_{k}\right)$, is denoted as $O_{i}^{g}(\Omega) \triangleq \mathcal{C}_{i}\left(\Omega^{g}\right)$ for the autonomous system $x_{k+1}=f\left(x_{k}, g\left(x_{k}\right)\right)$.

Remark 2.6. All the properties of controllable and admissible sets hold true for the corresponding sets of autonomous systems. Note that $O_{i}^{g}(\Omega)=\mathcal{K}_{i}\left(\Omega^{g}, \Omega^{g}\right)=\mathcal{K} O_{i}^{g}\left(\Omega, \Omega^{g}\right)$. These sets can be computed using Algorithms 2.1 and 2.2.

\subsubsection{Computing the Maximal Stabilisable Set:}

Algorithm 2.3 (Maximal stabilisable set). Algorithm 2.1 can be used to compute the i-step stabilisable set $\mathcal{S}_{i}(\Omega, \mathbb{T})$ contained in $\Omega$ by noting that

$$
\mathcal{S}_{i}(\Omega, \mathbb{T})=\mathcal{K}_{i}(\Omega, \mathbb{T})
$$

If $\mathcal{S}_{i}(\Omega, \mathbb{T})=\mathcal{S}_{i+1}(\Omega, \mathbb{T})$, then $\mathcal{S}_{\infty}(\Omega, \mathbb{T})=\mathcal{S}_{i}(\Omega, \mathbb{T})$.

Proposition 2.4. Some properties of stabilisable sets are:

(i) Each set $\mathcal{S}_{i}(\Omega, \mathbb{T})$ is control invariant;

(ii) Each set $\mathcal{S}_{i+1}(\Omega, \mathbb{T}) \supseteq \mathcal{S}_{i}(\Omega, \mathbb{T})$;

(iii) Each set $\mathcal{S}_{i}(\Omega, \mathbb{T})=\bigcup_{k=0}^{i} \mathcal{S}_{k}(\Omega, \mathbb{T})$;

(iv) If the state $x_{k} \in S_{i+1}(\Omega, \mathbb{T}) \backslash S_{i}(\Omega, \mathbb{T}) \neq \emptyset$, then there exists a control input which will drive the state to $S_{i}(\Omega, \mathbb{T})$ at the next time instant;

(v) If the state $x_{k} \in \mathcal{S}_{i+1}(\Omega, \mathbb{T}) \backslash \mathcal{S}_{i}(\Omega, \mathbb{T}) \neq \emptyset$, then there does not exist a sequence which will drive the system to $\mathbb{T}$ in $i$ steps or less.

Theorem 2.4. $\mathcal{S}_{\infty}(\Omega, \mathbb{T})$ is finitely determined if and only if $\exists i \in \mathbb{N}$ such that $\mathcal{S}_{i}(\Omega, \mathbb{T})=\mathcal{S}_{i+1}(\Omega, \mathbb{T})$.

\section{Model Predictive Control}

This section briefly introduces Nonlinear Model Predictive Control (NMPC) and proceeds to address some feasibility issues related with solving the NMPC problem. The proofs for the results are given in [9]. 
Problem 3.1 (NMPC Regulation Problem). Solve

$$
\min _{\pi_{k}^{N}} F\left(\hat{x}_{P \mid k}\right)+\sum_{i=0}^{P-1} L\left(\hat{x}_{i \mid k}, \hat{u}_{i \mid k}\right)
$$

subject to

$$
\begin{array}{lr}
\hat{x}_{l+1 \mid k}=f\left(\hat{x}_{l \mid k}, \hat{u}_{l \mid k}\right), & \hat{x}_{0 \mid k}=x_{k} \\
\hat{x}_{l \mid k} \in \mathbb{X}, \quad \hat{u}_{l \mid k} \in \mathbb{U}, & l=0, \ldots, P-1 \\
\hat{u}_{l \mid k}=h\left(\hat{x}_{l \mid k}\right), & l=N, \ldots, P-1 \\
\hat{x}_{P \mid k} \in \mathbb{T} \subseteq \mathbb{X} &
\end{array}
$$

The decision variable in the NMPC problem is the control sequence $\pi_{k}^{N}=\left\{\hat{u}_{0 \mid k}, \hat{u}_{1 \mid k}, \ldots, \hat{u}_{N-1 \mid k}\right\}$. The variables $N$ and $P$ are the control and prediction horizons, respectively, and it is assumed that $P \geq N \geq 0$. Note that if $P=N$, then constraint (12c) is removed. $\mathbb{T}$ is the terminal constraint set.

Since the optimisation is over a finite horizon, in the design of the terminal cost functional $F\left(\hat{x}_{P \mid k}\right)$ and the stage cost functional $L\left(\hat{x}_{i \mid k}, \hat{u}_{i \mid k}\right)$, it is (usually) assumed that $\hat{u}_{l \mid k}=$ $h\left(\hat{x}_{l \mid k}\right)$ is a locally stabilising control law defined on $\mathbb{X}$ that will be applied on the infinite horizon for $l \geq P$. It is assumed that $L(\cdot, \cdot)$ is a non-negative functional defined on $\mathbb{X} \times \mathbb{U}$ and $F(\cdot)$ is a non-negative functional defined on $\mathbb{X}$.

At each time instant $k$, the current state $x_{k}$ of the system is measured. The new control input to the system is the first element of the (not necessarily optimal) solution $\pi_{k}^{N^{*}}$ to Problem 3.1, i.e. $\kappa\left(x_{k}\right) \triangleq \hat{u}_{0 \mid k}^{*}$. Here $\kappa(x)$ implicitly defines the NMPC control law, the closed-loop system being given by $x_{k+1}=f\left(x_{k}, \kappa\left(x_{k}\right)\right)$. State measurement and control input calculation is repeated at the next time instant. It is assumed that $N, P, F, L, h$ and $\mathbb{T}$ are the design variables and that $f, \mathbb{X}$ and $\mathbb{U}$ are fixed. The aim of the control action is to regulate the states and control inputs to $(0,0)$.

\subsection{Nominal Feasibility}

It is assumed that the set of ordered pairs $\left(x_{k}, \pi_{k}^{N}\right)$, which satisfy the constraints in (12), is non-empty. The feasible set $\mathbb{X}_{F}$ is the set of states $x_{k}$ for which a feasible control sequence $\pi_{k}^{N}$ to the NMPC problem exists, i.e. $\mathbb{X}_{F}$ is the orthogonal projection of (12) onto the first coordinate:

$$
\mathbb{X}_{F}(\mathbb{T}, N, P) \triangleq\left\{x_{k}: \exists \pi_{k}^{N} \text { s.t. }\left(x_{k}, \pi_{k}^{N}\right) \text { satisfies }(12)\right\} \text {. }
$$

The NMPC regulation problem is said to be feasible at time $k$ if and only if $x_{k} \in \mathbb{X}_{F} \neq \emptyset$. Note that $\mathbb{X}^{\kappa}=\mathbb{X}_{F}$ by definition.

Theorem 3.1. The feasible set $\mathbb{X}_{F}(\mathbb{T}, N, P)$ of the $N M P C$ regulation problem is given by:

$$
\mathbb{X}_{F}(\mathbb{T}, N, P)=\mathcal{K}_{N}\left(\mathbb{X}, \mathcal{K} O_{P-N}^{h}(\mathbb{X}, \mathbb{T})\right) .
$$

Proof. From the constraints (12), the solution to the NMPC problem has to satisfy $\hat{x}_{l \mid k} \in \mathbb{X}$ and $\hat{u}_{l \mid k}=h\left(\hat{x}_{l \mid k}\right) \in \mathbb{U}, \forall l=$ $N, \ldots, P-1$, therefore $\hat{x}_{l \mid k} \in \mathbb{X}^{h}, \forall l=N, \ldots, P-1$. Additionally, it is required that $\hat{x}_{P \mid k} \in \mathbb{T}$, therefore $\hat{x}_{N \mid k} \in$ $\mathcal{K} O_{P-N}^{h}(\mathbb{X}, \mathbb{T})$. Furthermore, $\hat{x}_{l \mid k} \in \mathbb{X}$ and $\hat{u}_{l \mid k} \in \mathbb{U}$ for all $l=0, \ldots, N-1$. From the definition of controllable sets, these constraints can be satisfied if only if $x_{k}=\hat{x}_{0 \mid k} \in$ $\mathcal{K}_{N}\left(\mathbb{X}, \mathcal{K} O_{P-N}^{h}(\mathbb{X}, \mathbb{T})\right)$.
Due to the finite-horizon nature of NMPC, it is possible that a bad choice of design variables could result in a solution with $\hat{x}_{1 \mid k}^{*} \in \mathbb{X} \backslash \mathbb{X}_{F}$. This will result in an infeasible problem at the next time instant, even in the absence of disturbances. Additionally, if $\mathbb{X}_{F} \backslash \mathcal{C}_{\infty}(\mathbb{X}) \neq \emptyset$ it is possible that $\hat{x}_{1 \mid k}^{*} \in \mathbb{X}_{F} \backslash \mathcal{C}_{\infty}(\mathbb{X})$, which will result in $x_{k+1} \notin \mathcal{C}_{\infty}(\mathbb{X})$. Since there does not exist a control sequence which will satisfy the constraints if the state is outside the maximal control invariant set, the NMPC problem will become infeasible at some future time.

The use of soft constraints is one way of solving the infeasibility problem [13]. However, this is not necessarily the best approach. State constraints will be violated at some future time, even in the absence of disturbances, if the solution to the soft-constrained problem results in $\hat{x}_{1 \mid k}^{*} \in \mathbb{X} \backslash C_{\infty}(\mathbb{X})$. This section addresses the nominal feasibility issue by providing conditions on $N, P$ and $\mathbb{T}$ under which feasibility (and hence state constraint satisfaction) can be guaranteed for all time, without the need for soft constraints.

Definition 3.1 (Feasible for all time). The NMPC problem is feasible for all time $k \in \mathbb{N}$ if and only if the initial state $x_{0}$ belongs to the feasible set and all future evolutions of the state belong to the feasible set, i.e. $x_{k} \in \mathbb{X}_{F}, \forall k \in \mathbb{N}$

The first result follows from the discussion above and is a necessary and sufficient condition for guaranteeing that the NMPC problem is feasible for all time:

Theorem 3.2. The NMPC problem is feasible for all time if and only if $x_{0} \in \mathbb{X}_{F} \cap \mathcal{C}_{\infty}(\mathbb{X})$ and $\forall x_{k} \in \mathbb{X}_{F} \cap \mathcal{C}_{\infty}(\mathbb{X})$ the solution to the NMPC problem results in $\hat{x}_{1 \mid k}^{*} \in \mathbb{X}_{F} \cap \mathcal{C}_{\infty}(\mathbb{X})$.

Definition 3.2 (Strongly feasible). The NMPC problem is strongly feasible if and only if for all $x_{0} \in \mathbb{X}_{F}$ the NMPC problem is feasible for all time.

Theorem 3.3. (i) The NMPC problem is strongly feasible if and only if the feasible set is a positively invariant set for the closed-loop system $x_{k+1}=f\left(x_{k}, \kappa\left(x_{k}\right)\right)$; (ii) The NMPC problem is strongly feasible only if the feasible set is a control invariant set for the system $x_{k+1}=f\left(x_{k}, u_{k}\right)$.

Remark 3.1. $\mathbb{X}_{F}$ is a control invariant set only if $\mathbb{X}_{F}$ is a subset of the maximal control invariant set $C_{\infty}(\mathbb{X})$.

Note that control invariance is only a necessary condition for a strongly feasible NMPC problem. The design variables that determine whether $\mathbb{X}_{F}$ is control invariant are $N$, $P, h\left(x_{k}\right)$ and $\mathbb{T}$. However, all the design variables, including the cost functionals $F\left(x_{k}\right)$ and $L\left(x_{k}, u_{k}\right)$, determine whether $\mathbb{X}_{F}$ is positively invariant for the closed-loop system. The following new sufficient condition is a weaker result than the well-known "control invariant terminal set" condition:

Theorem 3.4. If $\mathbb{X}_{F}(\mathbb{T}, N, P)$ is control invariant, then the NMPC problem with a control horizon of $\tilde{N} \geq N+1$ and a prediction horizon of $\tilde{P}=P+\tilde{N}-N$ is strongly feasible.

Remark 3.2. This result implies that by increasing the control and prediction horizons by the same amount will result in a strongly feasible NMPC problem. This result holds even if $\mathbb{X}_{F}(\mathbb{T}, N-1, P-1)$ and/or $\mathbb{T}$ are not control invariant. 


\subsection{Equal Control and Prediction Horizons}

Note that the terminal controller $h\left(x_{k}\right)$ does not affect the feasible set if the control and prediction horizons are equal. The only design variables that determine the feasible set are the control horizon $N=P$ and the terminal constraint set $\mathbb{T}$.

3.2.1 Terminal Set $\mathbb{T}=\mathbb{X}$ : The following new result on the feasibility of the NMPC problem considers the case when the terminal state is equal to the state constraints.

Theorem 3.5. Let $P=N$ and $\mathbb{T}=\mathbb{X}$ :

(i) The feasible set is equal to the $N$-step admissible set, i.e. $\mathbb{X}_{F}(\mathbb{X}, N, N)=\mathcal{C}_{N}(\mathbb{X})$. The feasible set contains the maximal control invariant set, i.e. $\mathcal{C}_{\infty}(\mathbb{X}) \subseteq \mathbb{X}_{F}(\mathbb{X}, N, N)$. The feasible set is control invariant if and only if the maximal control invariant set is finitely determined and the control horizon is equal to or greater than its determinedness index $i^{*}$, i.e. $N \geq i^{*}$;

(ii) The NMPC problem is strongly feasible if the control horizon is larger than the determinedness index $i^{*}$ of the maximal control invariant set $\mathcal{C}_{\infty}(\mathbb{X})$, i.e. $N \geq i^{*}+1$;

(iii) A larger control horizon results in a smaller feasible set. The size of the feasible set stops decreasing if and only if the maximal control invariant set is finitely determined and the control horizon is larger than its determinedness index, i.e. $i^{*} \geq N_{1}>N_{2}$ if and only if $\mathbb{X}_{F}\left(\mathbb{X}, N_{1}, N_{1}\right) \subset \mathbb{X} F\left(\mathbb{X}, N_{2}, N_{2}\right)$. Furthermore, $\mathbb{X}_{F}(\mathbb{X}, N, N)=\mathcal{C}_{\infty}(\mathbb{X})=\mathcal{C}_{i^{*}}(\mathbb{X}), \forall N \geq i^{*}$.

Remark 3.3. For $\mathbb{T}=\mathbb{X}$ and $N=P$, Theorem 3.5 implies that one cannot choose the design variables such that the NMPC problem is strongly feasible if and only if the maximal control invariant set is not finitely determined. This is a problem, since in general one cannot guarantee finite determinedness or that the determinedness index will be small enough for the controller to be implementable. It might be possible that a redesign of the state and/or control constraints or the system might solve the determinedness problem.

3.2.2 Control Invariant Terminal Set: The following theorem contains the well-known control invariant terminal constraint condition, as discussed in [11]:

Theorem 3.6. Let $P=N$ and the terminal constraint set be a control invariant subset of $\mathbb{X}$, i.e. $Q(\mathbb{T}) \cap \mathbb{T}=\mathbb{T} \subseteq \mathbb{X}$ :

(i) The feasible set is equal to the $N$-step stabilisable set, i.e. $\mathbb{X}_{F}(\mathbb{T}, N, N)=\mathcal{S}_{N}(\mathbb{X}, \mathbb{T})$. Hence. the feasible set is control invariant and contained within the maximal control invariant set, i.e. $\mathbb{X}_{F}(\mathbb{T}, N, N) \subseteq \mathcal{C}_{\infty}(\mathbb{X})$;

(ii) The NMPC problem is strongly feasible;

(iii) A larger control horizon results in a larger feasible set. The size of the feasible set stops increasing if and only if the maximal stabilisable set is finitely determined and the control horizon is larger than its determinedness index $i^{*}$, i.e. $i^{*} \geq N_{1}>N_{2}$ if and only if $\mathbb{X}_{F}\left(\mathbb{T}, N_{1}, N_{1}\right) \supset \mathbb{X}_{F}\left(\mathbb{T}, N_{2}, N_{2}\right)$. Furthermore, $\mathbb{X}_{F}(\mathbb{T}, N, N)=\mathcal{S}_{\infty}(\mathbb{X}, \mathbb{T})=\mathcal{S}_{i^{*}}(\mathbb{X}, \mathbb{T}), \forall N \geq i^{*}$.

Corollary 3.1. If $N=P$, then the feasible set of an $N M P C$ problem with a control invariant terminal constraint set $\mathbb{T} \subseteq$ $\mathbb{X}$, is always contained within the feasible set of an NMPC problem with $\mathbb{T}=\mathbb{X}$, i.e. $\mathbb{X}_{F}(\mathbb{T}, N, N) \subseteq \mathbb{X}_{F}(\mathbb{X}, N, N)$.

\subsection{Different Control and Prediction Horizons}

When the prediction horizon is larger than the control horizon, feasibility analysis of the NMPC problem is slightly more involved.

Corollary 3.2. Assume that the maximal control invariant set is finitely determined with determinedness index $i^{*}$ and that $P>N$. If $\mathbb{T}$ is any subset of $\mathbb{X}$ and $P \geq i^{*}$, then $\mathbb{X}_{F}(\mathbb{T}, N, P) \subseteq \mathcal{C}_{\infty}(\mathbb{X})$.

Note that since Corollary 3.2 does not assume any invariance condition on $\mathbb{T}$, the result does not imply that the feasible set is control invariant. Even if $\mathbb{T}$ is control invariant, if $P>N$ one cannot guarantee in general that the NMPC problem is strongly feasible or even control invariant.

3.3.1 Terminal Set $\mathbb{T}=\mathbb{X}$ : The following lemma is useful in understanding Theorem 3.7:

Lemma 3.1. If $\mathbb{T}=\mathbb{X}^{h}$, then $\mathcal{K} O_{i}^{h}(\mathbb{X}, \mathbb{T})=O_{i}^{h}(\mathbb{X})$ for all $i \geq 0$. If $\mathbb{T} \supseteq \mathbb{X}^{h}$ and the maximal positively invariant set $O_{\infty}^{h}(\mathbb{X})$ is finitely determined with determinedness index $i^{*}$, then $\mathcal{K} O_{i}^{h}(\mathbb{X}, \mathbb{T})=O_{\infty}^{h}(\mathbb{X})$ for all $i \geq i^{*}+1$.

Theorem 3.7. Let $P>N$ and $\mathbb{T}=\mathbb{X}$ :

(i) The feasible set is equal to the $N$-step controllable set to $\mathcal{K} O_{P-N}^{h}(\mathbb{X}, \mathbb{X})$ for the closed-loop system $x_{k+1}=$ $f\left(x_{k}, h\left(x_{k}\right)\right)$, i.e. $\quad \mathbb{X}_{F}(\mathbb{X}, N, P)=\mathcal{K}_{N}\left(\mathbb{X}, \mathcal{K} O_{P-N}^{h}(\mathbb{X}, \mathbb{X})\right)$. Hence, the feasible set is not necessarily control invariant;

(ii) The NMPC problem is strongly feasible if the difference between the prediction and control horizons is larger than the determinedness index of the maximal positively invariant set $O_{\infty}^{h}(\mathbb{X})$, i.e. $P-N \geq i^{*}+1$. The condition relaxes to $P-N \geq i^{*}$ if $\mathbb{T}=\mathbb{X}^{h}$;

(iii) Assume that $N$ is fixed. A larger prediction horizon results in a smaller feasible set. The size of the feasible set stops decreasing if and only if the maximal positively invariant set $O_{\infty}^{h}(\mathbb{X})$ is finitely determined and the difference between the prediction and control horizons is larger than its determinedness index, i.e. $N+i^{*}+1 \geq P_{1}>P_{2}>N$ if and only if $\mathbb{X}_{F}\left(\mathbb{X}, N, P_{1}\right) \subset \mathbb{X}_{F}\left(\mathbb{X}, N, P_{2}\right)$. Furthermore, $\mathbb{X}_{F}(\mathbb{X}, N, P)=S_{N}\left(\mathbb{X}, O_{\infty}^{h}(\mathbb{X})\right), \forall P \geq N+i^{*}+1 ;$

(iv) Assume that $P \geq N+i^{*}+1$. A larger control horizon results in a larger feasible set. The size of the feasible set stops increasing if and only if the maximal stabilisable set $S_{\infty}\left(\mathbb{X}, O_{\infty}^{h}(\mathbb{X})\right)$ is finitely determined and the control horizon is larger than its determinedness index $j^{*}$, i.e. $j^{*} \geq N_{1}>N_{2}$ if and only if $\mathbb{X}_{F}\left(\mathbb{X}, N_{1}, P\right) \supset \mathbb{X}_{F}\left(\mathbb{X}, N_{2}, P\right)$. Furthermore, $\mathbb{X}_{F}(\mathbb{X}, N, P)=\mathcal{S}_{\infty}\left(\mathbb{X}, O_{\infty}^{h}(\mathbb{X})\right), \forall N \geq j^{*}, P \geq N+i^{*}+1$.

Note that if $P-N \leq i^{*}$, then one cannot guarantee that the feasible set is control invariant, except that there exists a subset of the feasible set which is control invariant. It is also difficult to say anything useful about the size of the feasible set w.r.t. the length of the horizons.

The following result is useful when the determinedness index of $O_{\infty}^{h}(\mathbb{X})$ is known, but $O_{\infty}^{h}(\mathbb{X})$ does not have a simple expression:

Corollary 3.3. If $O_{\infty}^{h}(\mathbb{X})$ is finitely determined with determinedness index $i^{*}$, then the feasible set of an NMPC problem with terminal constraint $\mathbb{T}=O_{\infty}^{h}(\mathbb{X})$ and $N=P$ is equal 
to the feasible set of an NMPC problem with $\mathbb{T}=\mathbb{X}$ and $P \geq N+i^{*}+1$, i.e. $\mathbb{X}_{F}\left(O_{\infty}^{h}(\mathbb{X}), N, N\right)=\mathbb{X}_{F}(\mathbb{X}, N, P)$ for all $P \geq N+i^{*}+1$. Both problems are strongly feasible.

3.3.2 Control Invariant Terminal Set: In general, if $\mathbb{T}$ is any control invariant subset of $\mathbb{X}$ and $P>N$, it is difficult to say anything about the feasibility of the NMPC problem. However, the following theorem is useful if $O_{\infty}^{h}(\mathbb{X})$ is complex and it is easy to obtain a simple expression for a positively invariant subset of $O_{\infty}^{h}(\mathbb{X})$ :

Theorem 3.8. Let $P>N$ and the terminal constraint set $\mathbb{T}$ be a positively invariant set for the closed-loop system $x_{k+1}=f\left(x_{k}, h\left(x_{k}\right)\right)$, hence it is also a control invariant set for the system $x_{k+1}=f\left(x_{k}, u_{k}\right)$, i.e. $O_{\infty}^{h}(\mathbb{T})=\mathbb{T} \subseteq O_{\infty}^{h}(\mathbb{X})$ :

(i) The feasible set is equal to the $N$-step stabilisable set to $\mathcal{K} O_{P-N}^{h}(\mathbb{X}, \mathbb{T})$, i.e. $\mathbb{X}_{F}(\mathbb{T}, N, P)=\mathcal{S}_{N}\left(\mathbb{X}, \mathcal{K} O_{P-N}^{h}(\mathbb{X}, \mathbb{T})\right)$. Furthermore, the feasible set is control invariant;

(ii) The NMPC problem is strongly feasible;

(iii) Assume that $N$ is fixed. A larger prediction horizon results in a larger feasible set. The size of the feasible set stops increasing if and only if $\mathcal{K} O_{\infty}^{h}(\mathbb{X}, \mathbb{T})$ is finitely determined and the difference between the prediction and the control horizon is equal to or greater than its determinedness index $i^{*}$, i.e. $N+i^{*} \geq P_{1}>P_{2}>N$ if and only if $\mathbb{X}_{F}\left(\mathbb{T}, N, P_{1}\right) \supset \mathbb{X}_{F}\left(\mathbb{T}, N, P_{2}\right)$. Furthermore, $\mathbb{X}_{F}(\mathbb{T}, N, P)=$ $S_{N}\left(\mathbb{X}, \mathcal{K} O_{\infty}^{h}(\mathbb{X}, \mathbb{T})\right), \forall P-N \geq i^{*}$;

(iv) Assume that $P-N$ is fixed. A larger control horizon results in a larger feasible set. The size of the feasible set stops increasing if and only if $\mathcal{S}_{\infty}\left(\mathbb{X}, \mathcal{K} O_{P-N}^{h}(\mathbb{X}, \mathbb{T})\right)$ is finitely determined and the control horizon is larger than its determinedness index $j^{*}$, i.e. $j^{*} \geq N_{1}>N_{2}$ if and only if $\mathbb{X}_{F}\left(\mathbb{T}, N_{1}, P\right) \supset \mathbb{X}_{F}\left(\mathbb{T}, N_{2}, P\right)$; Furthermore, $\mathbb{X}_{F}(\mathbb{T}, N, P)=\mathcal{S}_{\infty}\left(\mathbb{X}, \mathcal{K} O_{P-N}^{h}(\mathbb{X}, \mathbb{T})\right), \forall N \geq j^{*}$. Additionally, $\mathbb{X}_{F}(\mathbb{T}, N, P)=\mathcal{S}_{\infty}\left(\mathbb{X}, \mathcal{K} O_{\infty}^{h}(\mathbb{X}, \mathbb{T})\right.$ ) for all $N \geq s^{*}$ if $S_{\infty}\left(\mathbb{X}, \mathcal{K} O_{\infty}^{h}(\mathbb{X}, \mathbb{T})\right)=\mathcal{S}_{s^{*}}\left(\mathbb{X}, \mathcal{K} O_{\infty}^{h}(\mathbb{X}, \mathbb{T})\right.$ and $P-N \geq i^{*}$.

The conclusion that increasing the difference between the control and prediction horizon results in a larger feasible set, provided $\mathbb{T}$ is positively invariant for the system $x_{k+1}=$ $f\left(x_{k}, h\left(x_{k}\right)\right)$, is also reported in [5]. The idea of using different control and prediction horizons to reduce the computational burden in NMPC, is discussed in [15].

\section{Conclusions and Further Remarks}

The main contribution of this paper is the development of a formal framework for the analysis and synthesis of NMPC controllers with guaranteed nominal feasibility. The effect of the terminal constraint set and horizons on the invariance properties and size of the feasible set was discussed. Though some of the results presented are reasonably wellknown and can be applied immediately to linear systems, this paper showed that these ideas can easily be extended to nonlinear systems. Though the computation of the various sets are difficult for general nonlinear systems, some algorithms for piecewise affine and hybrid systems are being developed $[1,9,14]$.

Extensions of this work include conditions for guaranteeing robust feasibility [9]. Issues such as guaranteeing feasibility with output feedback, set-point tracking, time-varying systems and time-varying constraints can also be addressed using this framework [9]. As control systems become more complex and performance requirements more demanding, the application of invariant set theory will inevitably become a standard procedure in the design and implementation of constrained control schemes such as MPC.

\section{References}

[1] A. Bemporad, F.D. Torrisi, and M. Morari. Optimizationbased verification and stability characterization of piecewise affine and hybrid systems. In B.H. Krogh and N. Lynch, editors, Hybrid Systems: Computation and Control, Lecture Notes in Computer Science. Springer-Verlag, 2000.

[2] F. Blanchini. Ultimate boundedness control for uncertain discrete-time systems via set-induced lyapunov functions. IEEE Transactions on Automatic Control, 39(2):428-433, February 1994.

[3] F. Blanchini. Set invariance in control. Automatica, 35:1747-1767, 1999.

[4] A. Casavola, M. Giannelli, and E. Mosca. Global predictive regulation of null-controllable input-saturated linear systems. IEEE Transactions on Automatic Control, 44(11):22262230, November 1999.

[5] G. De Nicolao, L. Magnani, L. Magni, and R. Scattolini. A stabilizing receding horizon controller for nonlinear discrete time systems. In Proceedings of the American Control Conference, pages 270-271, Chicago IL, USA, June 2000.

[6] C.E.T. Doréa and J.C. Hennet. $(A, B)$-invariant polyhedral sets of linear discrete-time systems. Journal of Optimization Theory and Applications, 103(3):521-542, December 1999.

[7] E.G. Gilbert and K.T. Tan. Linear systems with state and control constraints: The theory and application of maximal output admissible sets. IEEE Transactions on Automatic Control, 36(9):1008-1020, September 1991.

[8] S.S. Keerthi and E.G. Gilbert. Computation of minimumtime feedback control laws for discrete-time systems with statecontrol constraints. IEEE Transactions on Automatic Control, AC32(5):432-435, 1987.

[9] E.C. Kerrigan. PhD thesis, University of Cambridge, UK, in preparation.

[10] I. Kolmanovsky and E.G. Gilbert. Theory and computation of disturbance invariant sets for discrete-time linear systems. Mathematical Problems in Engineering: Theory, Methods and Applications, 1998.

[11] D.Q. Mayne, J.B. Rawlings, C.V. Rao, and P.O.M. Scokaert. Constrained model predictive control: Stability and optimality. Automatica, 36:789-814, 2000.

[12] J.A. Primbs and V. Nevistić. Feasibility and stability of constrained finite receding horizon control. Automatica, 36:965-971, 2000.

[13] P.O.M. Scokaert and J.B. Rawlings. Feasibility issues in model predictive control. AIChE Journal, 45(8):1649-1659, August 1999.

[14] R. Vidal, S. Schaffert, J. Lygeros, and S. Sastry. Controlled invariance of discrete time systems. Technical Report UCB/ERL M99/65, Electrical Engineering and Computer Sciences, University of California at Berkeley, Berkeley, CA 94720-1774, USA, December 1999.

[15] A. Zheng and F. Allgöwer. Towards a practical nonlinear predictive control algorithm with guaranteed stability for largescale systems. In Proceedings of the American Control Conference, pages 2534-2538, Philadelphia, Pennsylvania, June 1998. 